\title{
Effect of surface tension on the behavior of adhesive contact based on Maugis-Dugdale model
}

\author{
Xinyao $\mathrm{Zhu}^{a, b}$, Wei $\mathrm{Xu}^{b, *}$ \\ ${ }^{a}$ Key Laboratory of Mechanism Theory and Equipment Design of Ministry of Education, Tianjin \\ University, Tianjin 300350, China \\ ${ }^{b}$ Faculty of Engineering and Physical Sciences, University of Surrey, Guilford GU2 7XH, UK \\ Corresponding author: Tel: +44 (0)1483 68 2368, E-mail: w.xu@surrey.ac.uk
}

\begin{abstract}
This present study reconsiders the effect of surface tension on the behavior of adhesive contact between a rigid sphere and an elastic half-plane, in which the adhesive interactions are supposed to follow the Dugdale laws. The adhesive contact issue is transformed into two inter coupled nonlinear integral equations which are governed by two parameters: $\lambda$ (Maugis adhesion parameter) and $S$ (elastocapillary number). By means of iteration method, numeric results are obtained. Analogous to the traditional Maugis-Dugdale (M-D) model, the results provide transition of the pull-off force between JKR and DMT type contact models through the Maugis adhesion parameter $\lambda$ with a fixed parameter $S$. On the other hand, with a fixed adhesion parameter $\lambda$, we also present the transitions of the pull-off force between four extreme models, named M-D, JKR, Bradley models and YoungDupre equation, through adjustment of the parameter $S$. Finally, we find the uniformity and discontinuity of the pressure distribution are affected by the combination of three factors: applied load $P$, adhesion parameter $\lambda$ and elastocapillary number $S$.
\end{abstract}

Keywords: Adhesive contact; surface tension; Dugdale law; Pull-off force; Discontinuity of pressure distribution.

\section{Introduction}

The contact of elastic bodies plays a significant role not only in nature living bodies but also in engineering systems. The first theory on non-adhesive contact mechanics of elastic bodies is ascribed to Hertz (1882), who discovered that the pressure in the contact region between two spheres exhibits an elliptical distribution. Bradley (1932) integrated the molecular forces between two rigid spheres and found the pull-off force is $-2 \pi R \Delta \gamma$, where $R$ is the reduced radius of curvature $(R=$ [1/R $\left.+1 / R_{2}\right]$, where $R_{1}$ and $R_{2}$ are the radii of curvature of the two spheres, respectively) and $\Delta \gamma$ is the work of adhesion which denotes the magnitude of work needed to separate two surfaces to infinity. Considering the effect of elasticity in the adhesive contact, Johnson et al. (1971, JKR) assumed that the adhesion forces are confined within the contact area in conjunction with the Hertzian contact pressure. The stresses on the contact perimeter are tensile and tend toward infinity, and the pull-off force is found to be $-1.5 \pi R \Delta \gamma$. Derjauin et al. (1975, DMT) considered the adhesion forces are produced over an annulus surrounding the contact region without affecting the surface profiles of the elastic contacting spheres, and resulting in the pull-off force equal $-2 \pi R \Delta \gamma$. Tabor (1977) proposed a parameter $\mu$ given by 


$$
\mu=\left(\frac{R \Delta \gamma^{2}}{E^{* 2} z_{0}^{3}}\right)^{\frac{1}{3}}
$$

to characterize the elastic behavior of adhesive contacts, where $E^{*}=\left[\left(1-v_{1}^{2}\right) / E_{1}+\left(1-v_{2}^{2}\right) / E_{2}\right]^{-1}$ is the effective Young's modulus, $\left(E_{1}, v_{1}\right.$ and $E_{2}, v_{2}$ denote the elastic modulus and Possion's ratio of the two contacting spheres, respectively), and $z_{0}$ represents the atomic equilibrium distance. $\mathrm{He}$ shows that the JKR and DMT theories represent two extremes of adhesive contacts: the JKR model holds for large and compliant spheres (high $\mu$ ), whereas the DMT model applies in small and stiff spheres (low $\mu$ ). By using the Dugdale law to describe the attractive forces, Maugis (1992) presented a more general theory to model the transition between the JKR and DMT model. In his theory, there is a uniform tensile stress $\left(\sigma_{0}\right)$ between two contacting spheres in an annular region $(a<r<c$, where $a$ and $c$ denote contact radius and adhesion radius respectively), just outside the contact area, where the two surfaces separation is less than $h_{0}$. The adhesive stress disappears outside this annular area. The work of adhesion in the Dugdale model is $\Delta y=\sigma_{0} h_{0}$, from which one can determine $h_{0}$. The tensile stress $\left(\sigma_{0}\right)$ and the work of adhesion $(\Delta \gamma)$ are chosen so that they equal the maximum tensile stress and the work of adhesion, respectively, of the Lennard-Jones potential law. With the assumption of parallel surfaces, $h_{0}$ is found to be $0.974 z_{0}$, which denotes the "equilibrium distance" between two parallel and flat surfaces at which the net local intermolecular force (obeying the Lennard-Jones potential law) is zero.

Among all the contact models reviewed above, the JKR model plays a significant role in the analysis of contact problems in the micro and nano scales. The JKR model has a substantial popularity in describing the adhesive contact of soft elastic spheres. However, in terms of the adhesive contact between hard mirco/nano particles and soft substrates, recent experiments exhibit a significant derivation from that predicted by JKR model. In fact, Style et al. (2013) found that the adhesion of small particle is analogous to the adsorption of particles at a liquid interface due to surface tension.

In order to interpret the effect of surface tension, Gurtin and Murdoch $(1975,1998)$ developed a 3D theory based on continuum mechanics concept considering the phenomenon of surface elasticity. For certain elementary deformation modes, there is good agreement between the prediction by Gurtin-Murdoch (GM) surface elasticity theory and experimental results and atomic simulations (Miller and Shenoy, 2000; Shenoy, 2002). Therefore, the GM surface elasticity theory enjoys a popularity in applications of the micromechanics to elucidate many size dependent phenomena at nanoscale, among which the effect of surface tension on non-adhesive contact is attracting increasing attention in recent years.

Pioneering studies of axisymmetric contact of an elastic half-plane with surface tension would be ascribed to Hajji (1978) about four decades ago, however, the surface tension effect on contact problem seems not to arise much attention until last decade. He and Lim (2006) formulated the 3D Green's function of an incompressible elastic half space with surface stress. Huang and $\mathrm{Yu}$ (2007) considered a half plane under concentrated normal or shear load by taking surface elasticity into consideration, and developed the solutions for the surface Green's functions by the Fourier integral transform technique. Wang and Feng (2007) investigated the response of a 2D elastic half space subject to a concentrated force, with constant surface tension and absence of surface elastic constants, and formulated the analytical solutions. By means of Stroh's formulism, Koguchi (2008) derived the surface Green's function of an anisotropic half space with surface effects, which was later demonstrated to coincide with the results by molecular dynamic analysis (Koguchi and Hayashi 
2009). Zhao and Rajapakse (2009) studied the $2 \mathrm{D}$ and axisymmetric loading problems of an elastic film by considering surface elasticity but without surface residual stress, and presented an analytical solution. Chen and Zhang (2010) developed the analytical Green's function for an isotropic elastic half-space subject to anti-plane shear deformation.

Since 2010, some studies begin to investigate the effects of surface tension against adhesive contact behavior. By using the thermos-dynamical approach, Salez et al. (2013) calculated the deformation of an elastic sphere on a rigid substrate under zero load, and established a continuous bridge between JKR model and Young-Dupre asymptotic regimes. Gao et al. (2014) investigated the effect of surface stress on the JKR model by employing the non-classical Boussinesq fundamental solutions, and found that the pull-off force increased while the critical contact radius decreased as a result of surface effect. Hui et al. (2015) studied the effect of surface tension on the non-slip adhesive contact between a rigid sphere and an incompressible substrate, and suggested a single dimensionless parameter: $\omega=\Gamma(G R)^{-2 / 3}(9 \pi \Delta \gamma / 4)^{-1 / 3}(\Gamma, G, R$ and $\Delta \gamma$ denote surface tension, shear modulus of the substrate, sphere radius and the interfacial work of adhesion respectively) to characterize the transition between JKR theory and Young-Dupre law. Long et al. (2016) reconsidered the same problem arisen by Hui et al. (2015), but without the requirement of non-slip and incompressibility of substrate material, and they presented an explicit relation between the contact radius and the indent depth in the absence of external load, which are more convenient in practical applications.

In all the studies mentioned in the last paragraph, the intermolecular forces are considered as short-range (In fact, the adhesive interactions are considered as a delta function in the JKR model), whereas the actual intermolecular forces are long-arrange. Very recently, Zhu and $\mathrm{Xu}$ (2018) investigated the effect of surface tension on the adhesive contact where the intermolecular forces obey the Lennard-Jones potential law, and presented some new features of adhesive contact behaviors with surface tension. In the present study, we consider that the adhesive interactions are modeled as Dugdale law in the adhesive contact with surface tension. The significance of this study is that it completes the investigation system of the adhesive contact mechanics with the existence of surface tension, as shown in Table 1. The objectives of this study mainly lie in three aspects: (1) to realize the transition between DMT model and JKR theory with the presence of surface tension; (2) to realize the transition between M-D model and Young-Dupre law as the surface tension strengthens; (3) to explore whether the results of these extreme model are dependent on the exact form of the intermolecular interaction potential, with the presence of surface tension.

\section{Table 1}

Summary of existing studies for adhesive and non-adhesive contact with and without surface tension.

\begin{tabular}{ccccc}
\hline & Non-adhesive & Delta function & L-J potential law & Dugdale Law \\
\hline No Surface tension & Hertz Model & JKR model & Greenwood & M-D model \\
& (Hertz, 1882) & (Johnson, et al., 1971) & $(1997)$ & Maugis (1992) \\
\hline Surface tension & Long and Wang & Hui et al. (2015) and & Zhu and Xu & The present study \\
& $(2013)$ & Long et al. (2016) & (2018) & \\
\hline
\end{tabular}

The contents in the first row represent the adopted model of interaction forces between two contacting surfaces.

\section{Formulation}

In the surface elasticity theory (Gurtin and Murdoch, 1975; Gurtin et al., 1998), the surface is 
treated as an inappreciably thin membrane ideally adhered to the bulk material. Although the elastic surface also has its equilibrium and constitutive equations analogous to classical theory of solid mechanics, they have some features due to the existence of surface stress. For an initially flat surface, the equilibrium conditions are given by (Chen et al., 2006; Mogilevskaya et al., 2011):

$$
\begin{gathered}
t_{\alpha}+\sum_{\beta=1}^{2} \sigma_{\beta \alpha, \beta}^{S}=0 \\
\sum_{i=1}^{3} \sum_{j=1}^{3} \sigma_{i j} n_{i} n_{j}=\sum_{\alpha=1}^{2} \sum_{\beta=1}^{2} \sigma_{\alpha \beta}^{S} \kappa_{\alpha \beta}
\end{gathered}
$$

where $t_{\alpha}(\alpha=1,2)$ is the surface traction in $x_{\alpha}$ direction, $n_{i}$ is the unit vector normal to the deformed surface, $\kappa_{\alpha \beta}$ is the curvature tensor of the surface and $\sigma_{\alpha \beta}{ }^{s}$ is the surface stress tensor. Cammarata (1994) presented the relationship between the surface stress tensor $\sigma_{\alpha \beta}{ }^{s}$ and surface energy density $\Gamma\left(\varepsilon_{\alpha \beta}\right)$, which could be considered as the constitutive equations for surface elasticity, as

$$
\sigma_{\alpha \beta}^{S}\left(\varepsilon_{\alpha \beta}\right)=\Gamma\left(\varepsilon_{\alpha \beta}\right) \delta_{\alpha \beta}+\frac{\partial \Gamma\left(\varepsilon_{\alpha \beta}\right)}{\partial \varepsilon_{\alpha \beta}}(4)
$$

where $\delta_{\alpha \beta}$ and $\varepsilon_{\alpha \beta}$ denote Kronecker delta function and surface strain tensor respectively. The second term of Eq. (4) denotes the dependence of the surface energy density on the elastic strain. If the change of the atomic spacing in deformation is infinitesimal, the contribution of the second term in Eq. (4) to the surface stress tensor is negligibly small compared to the surface tension (Yang, 2004; Shenoy, 2005), and hence one has

$$
\sigma_{\alpha \beta}^{s}=\Gamma\left(\varepsilon_{\alpha \beta}\right) \delta_{\alpha \beta}(5)
$$

Since atomic simulation indicates that the surface energy of nanoparticles remains almost constant when the radius of nanoparticles is larger than $4 \mathrm{~nm}$ (Bian et al., 2012), we assume that surface energy density $\Gamma$ is constant on the surface in the present study, resulting in a constant residual surface tension, which can capture the main surface effects in contact mechanics (Gao et al., 2013; Ardito et al., 2015). It is worth noting that the surface elasticity theory by Gurtin and Murdoch (1975) (G-M model) remains as an open topic, and later it is followed by subsequent new studies such as Huang and Wang (2006) who developed a hyperelastic surface model, and Chen and Yao (2014) who proposed the concept of surface energy density to characterize surface elasticity.

Based on the following hypotheses:

1. The surface tension $\Gamma$ in the membrane mentioned above is large enough such that it is not changed $^{1}$ appreciably after being subject to small deformations.

2. The surface is in frictionless contact with the bulk material.

Hajji (1978) developed an analytical solution for the normal stress $\sigma_{z}$ and displacement $(u, w)$ of a semi-infinite space subject to a vertical concentrated force $P$, with the foregoing surface tension:

$$
\begin{gathered}
\sigma_{z}(r, 0)=\frac{P}{2 \pi s}\left\{\frac{\pi}{2 s}\left[H_{0}\left(\frac{r}{s}\right)-Y_{0}\left(\frac{r}{s}\right)\right]-\frac{1}{r}\right\} \\
u(r, 0)=-\frac{(1-2 v) P}{4 \pi G} \int_{0}^{\infty} \frac{J_{1}(r t)}{1+s t} d t \\
w(r, 0)=\frac{P}{4 \Gamma}\left[H_{0}\left(\frac{r}{s}\right)-Y_{0}\left(\frac{r}{s}\right)\right](8)
\end{gathered}
$$

where $s=\Gamma(1-v) / G$, is an intrinsic material length indexing the relative significance of surface tension. $G$ and $v$ denote the shear modulus and Poisson's ratio of the half space respectively. $H_{n}(\cdot)$, $J_{n}(\cdot)$ and $Y_{n}(\cdot)$ denote the Struve function, Bessel function of the first kind and Bessel function of the

\footnotetext{
${ }^{1}$ For contact and adhesion analysis taking a variation of surface stresses into consideration, the readers are encouraged to refer the studies by Koguchi (2004, a and b)
} 
second kind, respectively, and $n$ is the order.

Now we begin to consider the adhesive contact of a rigid sphere with radius $R$ indenting on an elastic half-plane, as illustrated in Fig. 1. The half space is covered by a membrane with surface tension, and the adhesive interaction between the sphere and half-plane obeys obeying the Dugdale laws. In this regard, the contact pressure $p(r)$ is given by

$$
p(r)=\left\{\begin{array}{c}
-\sigma_{0}(a \leq r \leq c) \\
0(r>c)
\end{array}\right.
$$

where $a$ and $c$ denote contact radius and adhesion radius, respectively.

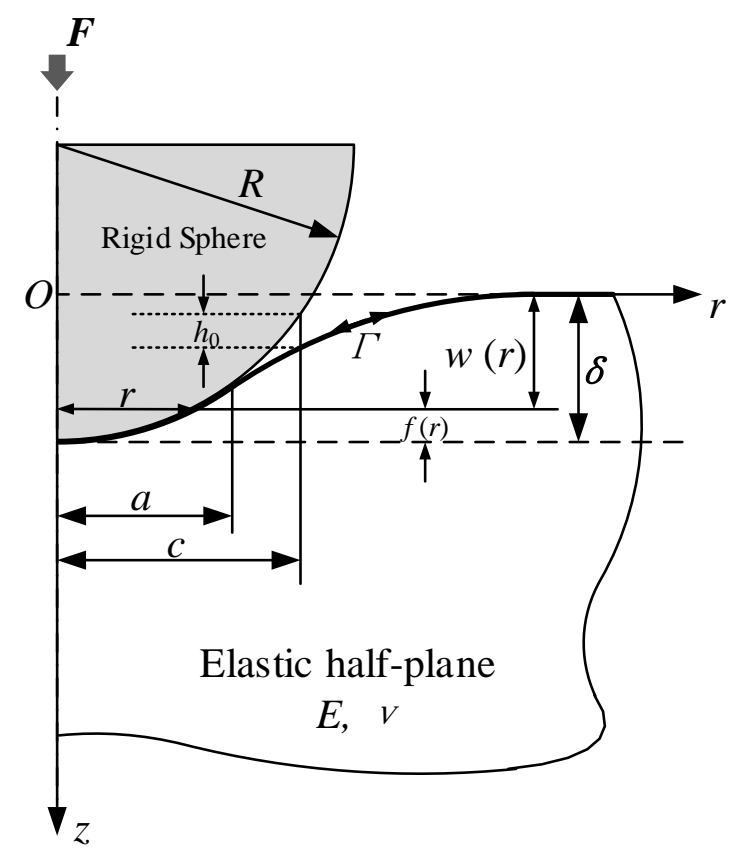

Fig. 1. Indentation of an elastic half-plane with surface tension by a rigid sphere. The contact pressure outside the contact area $(r>a)$ follows the Dugdale laws.

According to the geometrical relation in Fig.1, the displacement boundary condition in the contact area is given by

$$
w(r, 0)=\delta-f(r)(r \leq a)(10)
$$

where $w(r, 0), \delta$ and $f(r)$ denote the vertical deflection of the surface, the displacement of the rigid indenter in the $z$-direction and the axisymmetric shape of the indenter, respectively. For the spherical indenter geometry in Fig. 1, $f(r)=r^{2} / 2 R$ holds under the assumption of small deformation, i.e. $a<<$ $R$, where $R$ is the radius of the spherical indenter.

Fig. 2 illustrates the plan view of the pressure distribution in the contact region by means of polar coordinate system. According to Eq. (8), the normal displacement $d w(r, 0)$ at an arbitrary point $B(r$, 0 ) ascribed to the pressure $p(t)$ acting on an infinitesimal surface element of area $t d \theta d t$ at another arbitrary point $C(t, \theta)$ is given by:

$$
d w(r, 0)=\frac{p(t) t d \theta d t}{4 \Gamma} \psi_{0}\left(\frac{l}{s}\right)
$$

where $l=\left(r^{2}+t^{2}-2 r t \cos \theta\right)^{1 / 2}, \psi_{0}(x)=H_{0}(x)-Y_{0}(x)$. Thus the total normal displacement at point $B$ due to the pressure acting on the whole surface should be: 


$$
w(r, 0)=\int_{\theta=0}^{\pi} \int_{t=0}^{c} \frac{t p(t)}{2 \Gamma} \psi_{0}\left(\frac{l}{s}\right) d \theta d t
$$

Inserting Eq. (12) into Eq. (10) yields:

$$
\int_{\theta=0}^{\pi} \int_{t=0}^{c} \frac{t p(t)}{2 \Gamma} \psi_{0}\left(\frac{l}{s}\right) d \theta d t=\delta-\frac{r^{2}}{2 R}(0 \leq r \leq a)(13)
$$

Differentiating Eq. (13) with respect to $r$ leads to

$$
\frac{1}{2 \Gamma s} \int_{\theta=0}^{\pi} \int_{t=0}^{c} \psi_{1}\left(\frac{l}{s}\right) \frac{r-t \cos \theta}{l} p(t) t d \theta d t=\frac{r}{R}(0 \leq r \leq a)
$$

where $\psi_{1}(x)=H_{1}(x)-2 / \pi-Y_{1}(x)$. Since the contact pressure outside the contact region obeys the Dugdale laws, substituting Eq. (9) into (14), one has:

$\frac{1}{2 \Gamma s} \int_{\theta=0}^{\pi} \int_{t=0}^{a} \psi_{1}\left(\frac{l}{s}\right) \frac{r-t \cos \theta}{l} p(t) t d \theta d t-\frac{1}{2 \Gamma s} \int_{\theta=0}^{\pi} \int_{t=a}^{c} \psi_{1}\left(\frac{l}{s}\right) \frac{r-t \cos \theta}{l} \sigma_{0} t d \theta d t=\frac{r}{R}(0 \leq r \leq a)$

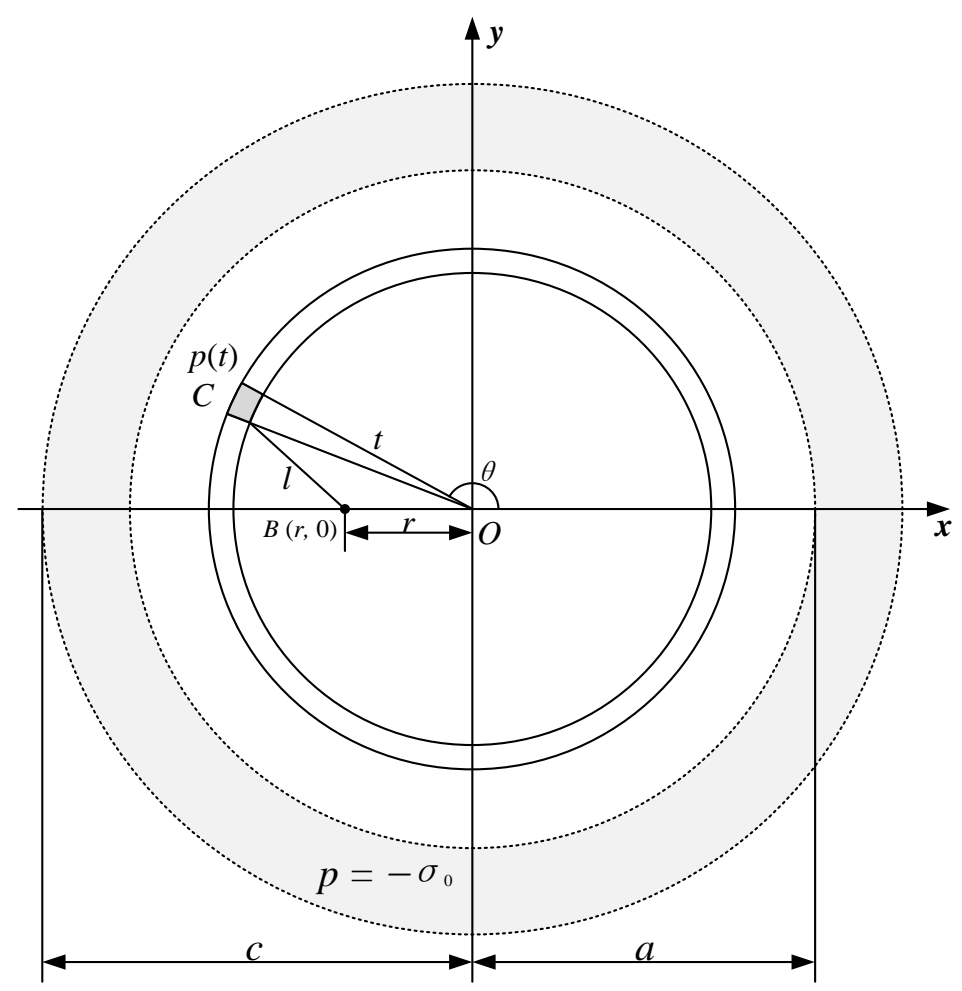

Fig. 2. Schematic of the planform of the adhesive contact, where the shaded annulus denotes the area $a<r<c$ with uniform pressure $-\sigma_{0}$.

For a given contact radius $a$, Eq. (15) is not sufficient to obtain the adhesion radius $c$ since the contact pressure within the contact region is unknown. Therefore, in order to complete the solution, we need one more equation where no new unknown is involved. Considering the geometrical relation for the gap of the contacting bodies at $r=c$ (adhesion radius), one has:

$$
w(c, 0)-w(0,0)=h_{0}-\frac{c^{2}}{2 R}(16)
$$

By letting $r=0$ and $c$ in Eq. (12), one can obtain the vertical deflections corresponding to these two 
points concerned. Inserting the vertical deflections of these two points into Eq. (16) leads to $\frac{1}{2 \Gamma} \int_{\theta=0}^{\pi} \int_{t=0}^{a} \psi_{0}\left(\frac{\sqrt{c^{2}+t^{2}-2 c t \cos \theta}}{s}\right) t p(t) d t d \theta-\frac{1}{2 \Gamma} \int_{\theta=0}^{\pi} \int_{t=a}^{c} \psi_{0}\left(\frac{\sqrt{c^{2}+t^{2}-2 c t \cos \theta}}{s}\right) t \sigma_{0} d t d \theta-$ $\frac{1}{2 \Gamma} \int_{\theta=0}^{\pi} \int_{t=0}^{a} \psi_{0}\left(\frac{t}{s}\right) t p(t) d t d \theta+\frac{1}{2 \Gamma} \int_{\theta=0}^{\pi} \int_{t=a}^{c} \psi_{0}\left(\frac{t}{s}\right) t \sigma_{0} d t d \theta=h_{0}-\frac{c^{2}}{2 R}(17)$

Making the following normalizations ${ }^{2}$

$$
s^{\prime}=\frac{s}{a}, \rho=\frac{r}{a}, p^{\prime}=\frac{p}{\sigma_{0}}, \tau=\frac{t}{a}, m=\frac{c}{a}, S=s\left(\frac{K}{\pi \Delta \gamma R^{2}}\right)^{\frac{1}{3}}, A=a\left(\frac{K}{\pi \Delta \gamma R^{2}}\right)^{\frac{1}{3}}, \lambda=2 \sigma_{0}\left(\frac{R}{\pi \Delta \gamma K^{2}}\right)^{\frac{1}{3}}
$$

where $K=8 G /[3(1-v)]$ and $m$ denotes the ratio of adhesion radius to contact radius, Eqs. (15) and (17) can be rewritten as

$$
\int_{\tau=0}^{1} G_{1}\left(\rho, \tau, s^{\prime}\right) p^{\prime}(\tau) d \tau-\int_{\tau=1}^{m} G_{1}\left(\rho, \tau, s^{\prime}\right) d \tau=\frac{3 \rho S^{2}}{2 A \lambda}(0 \leq \rho \leq 1)
$$

and

$\int_{\tau=0}^{1} G_{0}\left(\frac{m}{s^{\prime}}, \frac{\tau}{s^{\prime}}\right) \tau p^{\prime}(\tau) d \tau-\int_{\tau=1}^{m} G_{0}\left(\frac{m}{s^{\prime}}, \frac{\tau}{s^{\prime}}\right) \tau d \tau-\int_{\tau=0}^{1} G_{0}\left(0, \frac{\tau}{s^{\prime}}\right) \tau p^{\prime}(\tau) d \tau+\int_{\tau=1}^{m} G_{0}\left(0, \frac{\tau}{s^{\prime}}\right) \tau d \tau=$ $\frac{3 S}{\pi(\lambda A)^{2}}-\frac{3 m^{2} S}{4 \lambda}$

respectively, where

$$
G_{0}(x, y)=\int_{\theta=0}^{\pi} \psi_{0}\left(\sqrt{x^{2}+y^{2}-2 x y \cos \theta}\right) d \theta(21 a)
$$

and

$$
G_{1}(x, y, z)=y \int_{\theta=0}^{\pi} \psi_{1}\left(\frac{\sqrt{x^{2}+y^{2}-2 x y \cos \theta}}{z}\right) \frac{(x-y \cos \theta)}{\sqrt{x^{2}+y^{2}-2 x y \cos \theta}} d \theta
$$

Considering the combination of Eqs. (19) and (20), it can be seen that, for a given value of $m$, there are two unknowns, i.e. $A$ and $p^{\prime}$, and thus they could be solved. Once the contact pressure $p(r)$ is obtained, the total force $P$ can be calculated by integrating the pressure with respect to the adhesion radius $c$. If $P$ is scaled by $\pi R \Delta \gamma$, one has:

$$
\frac{P}{\pi R \Delta \gamma}=\pi \lambda A^{2} \int_{\tau=0}^{1} \tau p^{\prime}(\tau) d \tau-\frac{m^{2}-1}{2}
$$

On the other hand, the penetration $\delta$ can be obtained by letting $r=0$ in Eq. (13). Following the convention in the M-D model where $\delta$ is scaled by $\left(\pi^{2} \Delta \gamma^{2} R / K^{2}\right)^{1 / 3}$, one gets

$$
\frac{\delta}{\left(\frac{\pi^{2} \Delta \gamma^{2} R}{K^{2}}\right)^{1 / 3}}=\frac{2 \pi \lambda A^{2}}{3 S}\left[\int_{\tau=0}^{1} \psi_{0}\left(\frac{\tau}{s^{\prime}}\right) \tau p^{\prime}(\tau) d \tau-\int_{\tau=1}^{m} \psi_{0}\left(\frac{\tau}{s^{\prime}}\right) \tau d \tau\right]
$$

\section{Computational details}

For the facilitation of numeric computation, the non-linear integral Eqs. (19) and (20) need to be discretized first. The integrating ranges $[0,1]$ in Eqs. (19) and (20) are inserted by $N+1$ nodes with equal distance, resulting in $N$ intervals. Analogously, $M+1$ nodes are evenly placed in the integrating range $[1, m]$ in Eqs. (19) and (20) leading to $M$ segments. Therefore, Eqs. (19) and (20) can be discretized as

$$
\frac{1}{N} \sum_{j=1}^{N} G_{1}\left(\rho_{i}, \tau_{j}, s^{\prime}\right) p_{j}^{\prime}-\frac{m-1}{M} \sum_{j=N+1}^{M+N} G_{1}\left(\rho_{i}, \tau_{j}, s^{\prime}\right)=\frac{3 \rho_{i} S^{2}}{2 A \lambda}
$$

and

\footnotetext{
2 The typical value for $\sigma_{0}, R, \Delta \gamma, E^{*}$ and $\Gamma$ are $20 \mathrm{MPa}, 1 \mu \mathrm{m}, 10 \mathrm{~mJ} / \mathrm{m}^{2}, 1 \mathrm{MPa}$ and $72 \mathrm{~mJ} / \mathrm{m}^{2}$ (Style et al., 2013)
} 
$\frac{1}{N} \sum_{j=1}^{N}\left[G_{0}\left(\frac{m}{s^{\prime}}, \frac{\tau_{j}}{s^{\prime}}\right)-G_{0}\left(0, \frac{\tau_{j}}{s^{\prime}}\right)\right] \tau_{j} p_{j}^{\prime}-\frac{m-1}{M} \sum_{j=N+1}^{M+N}\left[G_{0}\left(\frac{m}{s^{\prime}}, \frac{\tau_{j}}{s^{\prime}}\right)-G_{0}\left(0, \frac{\tau_{j}}{s^{\prime}}\right)\right] \tau_{j}=\frac{3 s}{\pi(\lambda A)^{2}}-\frac{3 m^{2} s}{4 \lambda}$

respectively.

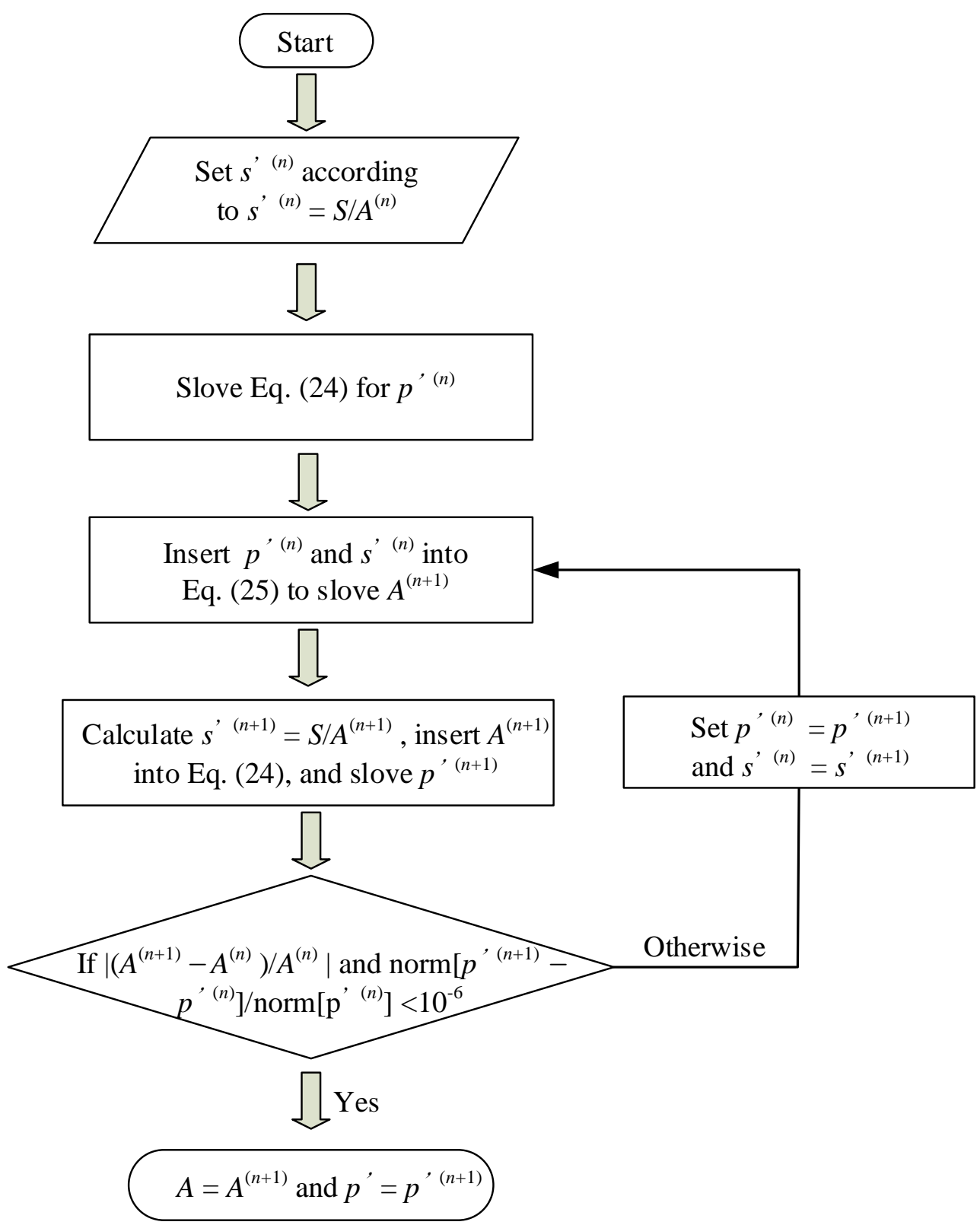

Fig. 3. An iterative scheme for solving the coupled nonlinear integral Eqs. (24) and (25).

In order to solve the coupled Eqs. (19) and (20) ( $A$ and $p_{j}^{\prime}$ are unknowns), iteration scheme is used as shown in Fig. 3 to obtain the solutions for $p$ ' and $A$. It can be seen that both Eqs. (19) and (20) are governed by the two parameters $\lambda$ and $S$. Before the implement of iteration, the adhesion parameter $\lambda$ and the parameter $S$ are fixed. For a given value of $m$, an initial guess for $A$ is made according to the Maugis-Dugdale model:

$$
\frac{\lambda A^{2}}{2}\left[\left(m^{2}-2\right) \csc ^{-1} m+\sqrt{m^{2}-1}\right]+\frac{4 \lambda^{2} A}{3}\left[\sqrt{m^{2}-1} \sec ^{-1} m-m+1\right]=1
$$


Once the iteration is completed, substitution of $p^{\prime}$ and $A$ into Eqs. (22) and (23) will determine both $P$ and $\delta$ respectively. For a new value $(m+d m)$, instead of the $A$ value determined by Eq. (26), we used the contact radius $A$ corresponding to $m$ as the initial guess which always proves to be closer to the solution.

The function $G_{0}(x, y)$ and $G_{1}(x, y, z)$ exhibit singularity where $x=y$ and $\theta \rightarrow 0$. In this paper, we adopt especial method to deal with this issue which is presented in the Appendix.

\section{Results and discussion}

In this study, the number of the nodes inserted into the integrating ranges $[0,1]$ and $[1, m]$ are the same with each other, i.e. $M=N=501$, which concurrently generates 500 even sections in both integrating ranges. Furthermore, our computational results indicate that the values of both $p$ ' and $A$ are insensitive to the node number after $M$ and $N$ are larger than 501 . To achieve convergence, the necessary iteration number depends on the value of $m$. When $A$ is low, $m$ is very high, a large iteration number is needed to achieve convergence. However, as $A$ increases corresponding to a smaller $m$ approaching unity, less iterations are required to meet the same convergence criterion shown in the diamond frame in Fig. 3. Throughout this present study, the results are confined to the adhesive contact between a rigid sphere and an elastic half-plane with surface tension. However, these results can always be expanded to other cases, e.g. contact between two elastic spheres or an elastic sphere and a rigid plane even in the presence of surface tension (Ding et al., 2015), providing the size of the sphere is much larger than the contact radius.

\subsection{The DMT model with surface tension}

In order to facilitate the transition from the DMT to JKR model under surface tension, it is imperative to introduce both models first. Hereafter, the DMT and JKR models with surface tension are named as ST-DMT and ST-JKR respectively. Although the ST-JKR has already been developed by Hui et al. (2015) and Long and Wang (2016), the ST-DMT model has not been yet completed. In this regard, the present section is dedicated to the development of the ST-DMT model.

Analogous to the derivation of the traditional DMT model, we first find the solution of Hertz contact model with surface tension. According to Eq. (13), the normal displacement $w(r, 0)$ of the surface in non-adhesive contact situation is

$$
w(r, 0)=\int_{\theta=0}^{\pi} \int_{t=0}^{a} \frac{p(t) t}{2 \Gamma} \psi_{0}\left(\frac{l}{s}\right) d \theta d t
$$

Within the contact area, we have

$$
w(r, 0)=\delta-\frac{r^{2}}{2 R}(0 \leq r \leq a)
$$

Inserting Eq. (27) into (28) and differentiating Eq. (28) with respect to $r$ lead to

$$
\frac{1}{2 \Gamma s} \int_{t=0}^{a} p(t) d t \int_{\theta=0}^{\pi} \psi_{1}\left(\frac{l}{s}\right) \frac{r-t \cos \theta}{l} t d \theta=\frac{r}{R}(0 \leq r \leq a)
$$

For a given value of $a$, Eq. (29) could be solved in terms of the contact pressure $p(t)$ within the contact region. Substituting $p(t)$ into Eq. (27) and letting $r=0$, we can obtain the penetration $\delta$. The total applied force $P$ is calculated by integrating the obtained pressure with respect to the whole surface. In fact, the Guass-Chebyshev quadrature formula has been adopted to solve Eq. (29) by Long and Wang (2013). Herewith, we skip all the details and only present the final solution. Based on the dimension analysis and the numerical results, we can parameterize the load $P$ and the contact 
radius $a$ in terms of the indent depth $\delta$ as (Long et al., 2017)

$$
P=K \sqrt{R \delta^{3}}\left[1+0.786\left(\frac{\sqrt{R \delta}}{s}\right)^{-0.851}\right](30 a)
$$

and

$$
a=\sqrt{R \delta}\left[\frac{1+0.445\left(\frac{\sqrt{R \delta}}{s}\right)^{-0.646}}{1+1.79\left(\frac{\sqrt{R \delta}}{s}\right)^{-0.757}}\right]^{\frac{1}{2}} \quad(30 b)
$$

respectively. Thus, in the ST-DMT model, the dependence of load $P$ on the indent depth $\delta$ is given by

$$
P=K \sqrt{R \delta^{3}}\left[1+0.786\left(\frac{\sqrt{R \delta}}{s}\right)^{-0.851}\right]-2 \pi R \Delta \gamma \quad \text { (31) }
$$

where the pull-off force is $-2 \pi R \Delta \gamma$, which will be justified in the next sub-section.

\subsection{The transition from the ST-DMT to ST-JKR model with surface tension}

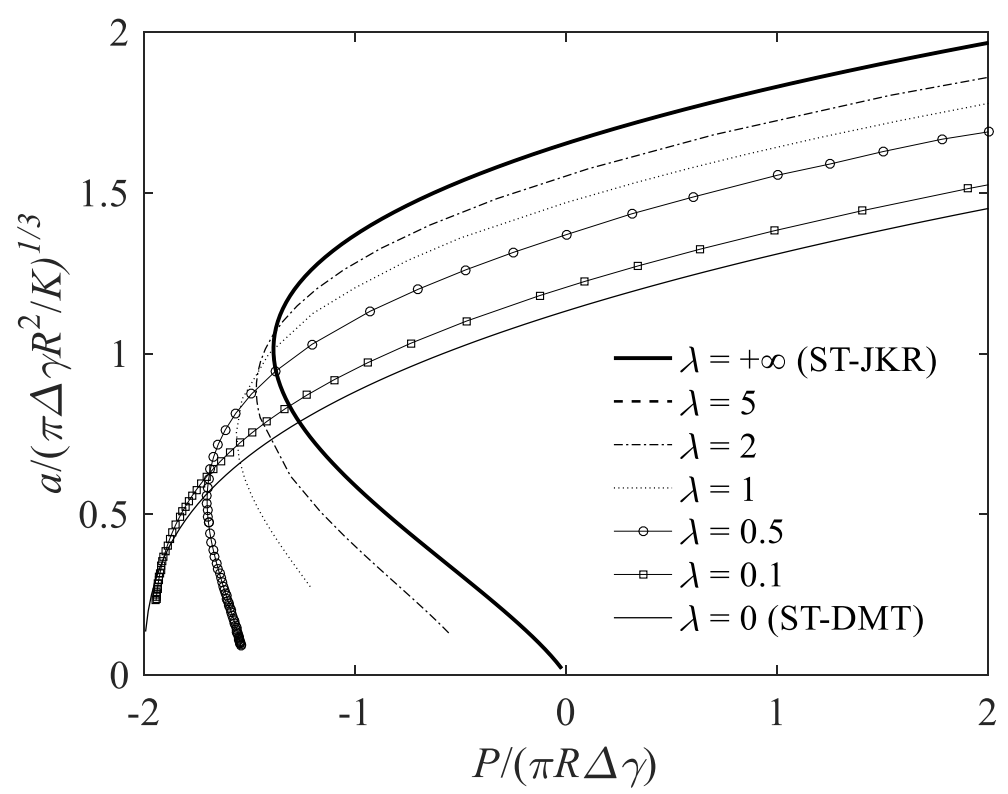

(a) 


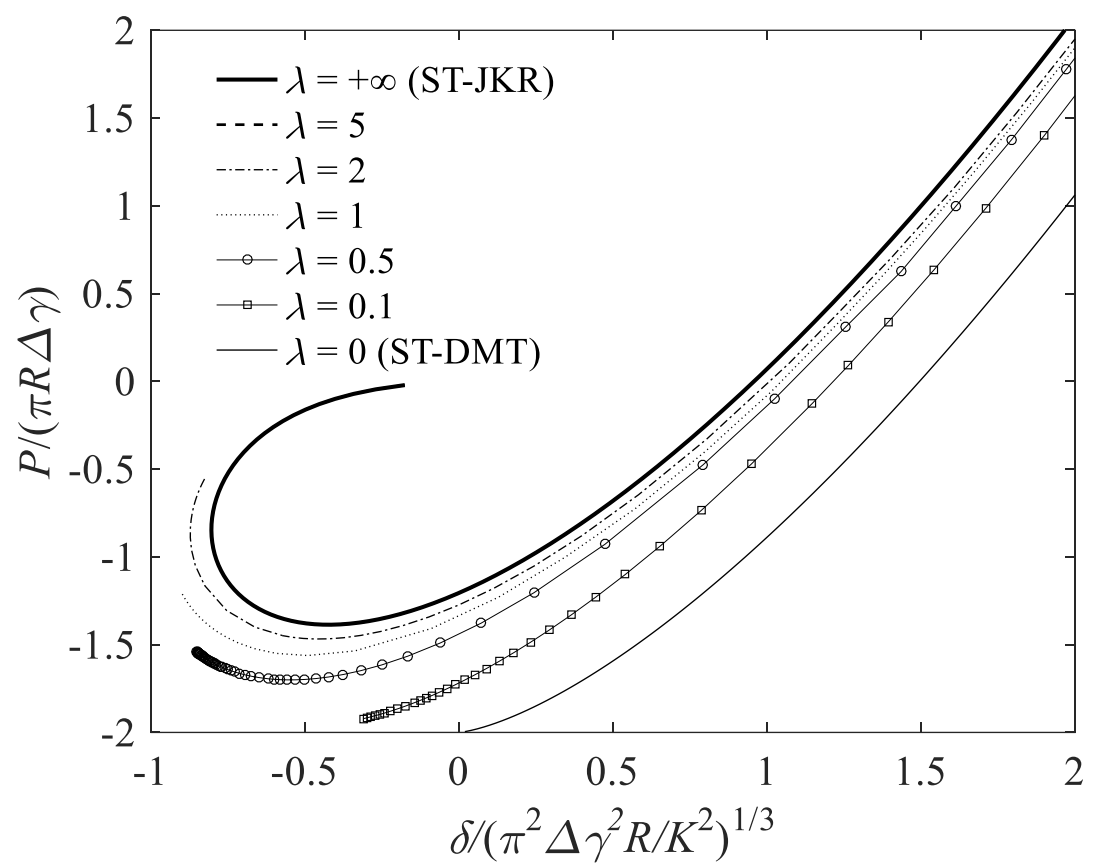

(b)

Fig. 4. (a) The variation of the dimensionless contact radius with the dimensionless normal force for various $\lambda$ values, where $S=0.1$ and $(b)$ the variation of the dimensionless normal force with the dimensionless penetration depth for various $\lambda$ values, with $S=0.1$. Note that the ST-JKR solution and the solution for $\lambda=5$ overlap each other.

In Fig. 4(a) the variations of the dimensionless contact radius, $a /\left(\pi \Delta \gamma R^{2} / K\right)^{1 / 3}$, with the dimensionless normal force, $P /(\pi \Delta \gamma R)$, are given as various values of the adhesion parameter $\lambda$. Similarly, Fig. $4(b)$ shows the variation of the dimensionless normal force, $P /(\pi \Delta \gamma R)$, with the dimensionless penetration depth, $\delta /\left(\pi^{2} \Delta \gamma^{2} R / K^{2}\right)^{1 / 3}$, as the same $\lambda$ values. The parameter $S$ is fixed $(S$ $=0.1$ ) in these two figures. The pull-off force is determined by the horizontal tangents of the lowest point in the force vs penetration depth curves. It can be seen from the Figs. 4(a) and 4(b), with the presence of surface tension, the contact behavior transmits from the ST-DMT model to the ST-JKR model as the adhesion parameter $\lambda$ increases from 0.1 to 5 , which is analogous to the situation in the traditional M-D model. It is particularly noteworthy that there is a superposition between the STJKR solution and the solution for $\lambda=5$, which means under $S=0.1$, our developed model can approximate the ST-JKR model. It is worth our attention that the presence of surface tension significantly affects the JKR limit, i.e. the pull-off differs from $1.5 \pi \Delta \gamma R$ which has already been interpreted by Hui et al. (2015).

Similarly, when the adhesion parameter is small enough, the ST-DMT limit is approached which, for a homogeneous half-plane without surface tension, corresponds to a pull-off force of $2 \pi \Delta \gamma R$. The presence of surface tension almost has no effect on this limit which is elucidated as follows. The DMT model is applicable in the case of the contact between a small rigid sphere and a stiff halfplane (low adhesion parameter $\lambda$ ). The traditional DMT model assumes the profiles of the contacting surfaces outside the contact region are not affected by the adhesive forces. The pull-off force corresponds to the instant when the contact radius is infinitesimal. At this moment, the surface of the half-plane remains unaffected, i.e. it is flat. Once the surface tension is involved, the situation could be regarded as the half-plane is covered with a membrane as mentioned in Section 2. This 
membrane would help the surface of the half-plane to retain its original profile, i.e. still a flat plane. On the other hand, the interaction energy per unit area between the two contacting surfaces are unaffected by the presence of the surface tension. Therefore, it is rational to assume that the pull-off force remains the same in the ST-DMT model as that of the traditional DMT model.

4.3 Pull-off force and transition between the M-D model and Young-Dupre law

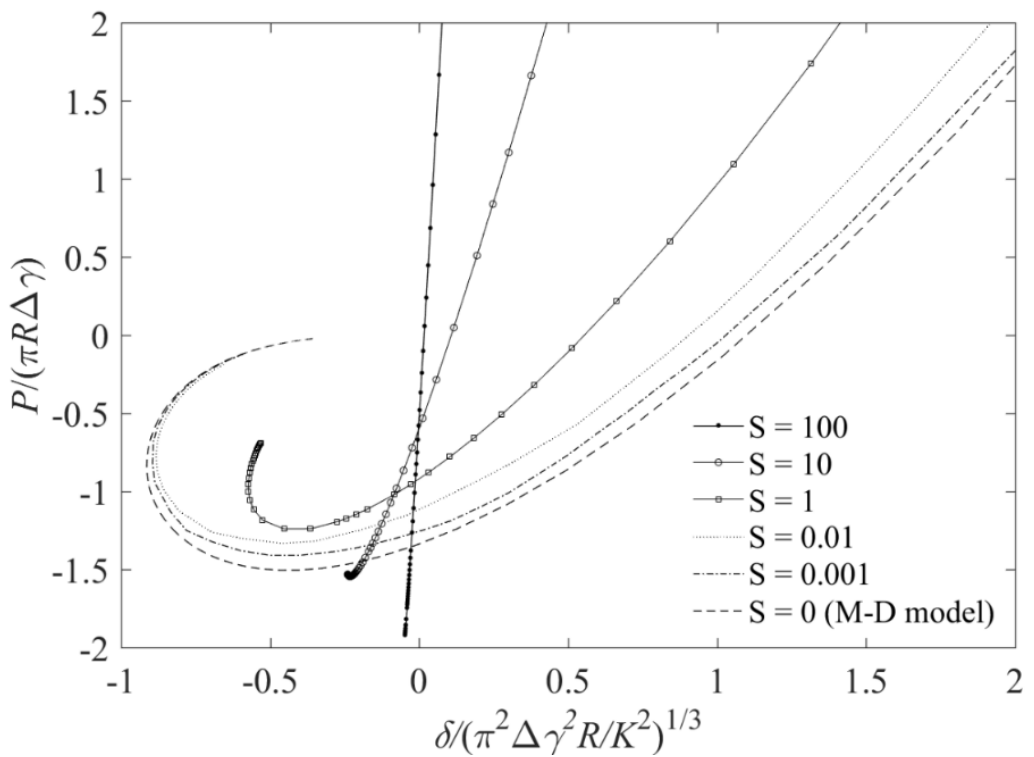

Fig. 5. The variation of the dimensionless normal force with the dimensionless penetration depth for various $S$ values with fixed $\lambda=5.0$.

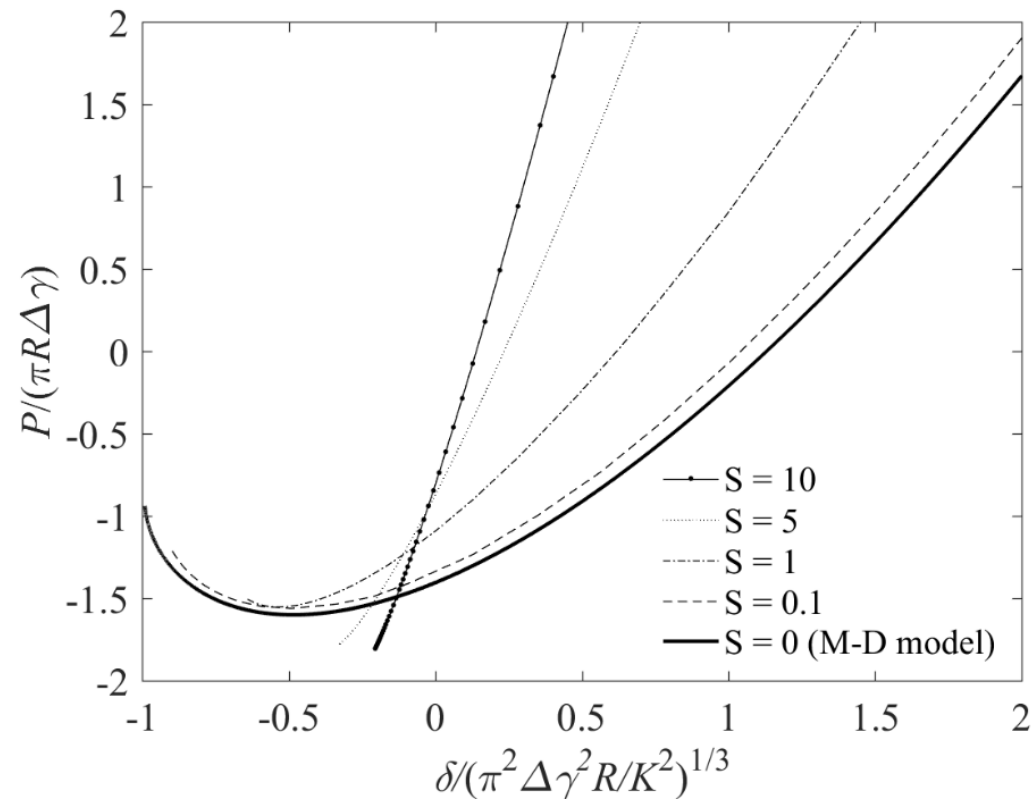

Fig. 6. The variation of the dimensionless normal force with the dimensionless penetration depth for various $S$ values with fixed $\lambda=1.0$. 
Fig. 5 and Fig. 6 illustrate the variations of the dimensionless normal force, $P /(\pi \Delta \gamma R)$, with the dimensionless penetration depth, $\delta /\left(\pi^{2} \Delta \gamma^{2} R / K^{2}\right)^{1 / 3}$, for various $S$ values. Fig. 5 corresponds to a large adhesion parameter $(\lambda=5.0)$, whereas Fig. 6 represents a small adhesion parameter $(\lambda=1.0)$. It is worth noting that at a high $S$ (e.g. $S=100$ in Fig. 5 and $S=10$ in Fig. 6), the load-approach curve below $P / \pi \Delta \gamma R=0$ almost exhibits linearity. In fact, this linearity resembles the Bradley model where the Lennard-Jones potential law is replaced by the Dugdale model. Videlicet, if the half-plane is subject to high surface tension, the adhesive contact behavior actually simulates that between two rigid spheres regardless of very strong adhesive forces (i.e. high value of $\lambda$ ), as shown by the thin solid curve in Fig. 5 where $\lambda=5$ and $S=100$. This similarity makes sense, because in an extreme case where an elastic half-plane is covered with a rigid membrane, i.e. $S \rightarrow+\infty$, any deformation of the surface is restricted as if the half-plane is rigid. On the other hand, with a small value of $S$ (e.g. $S=0.001$ ), i.e. the surface tension is weak, the load-approach curve approaches that of the M-D model as shown in Figs. 5 and 6, as expected.

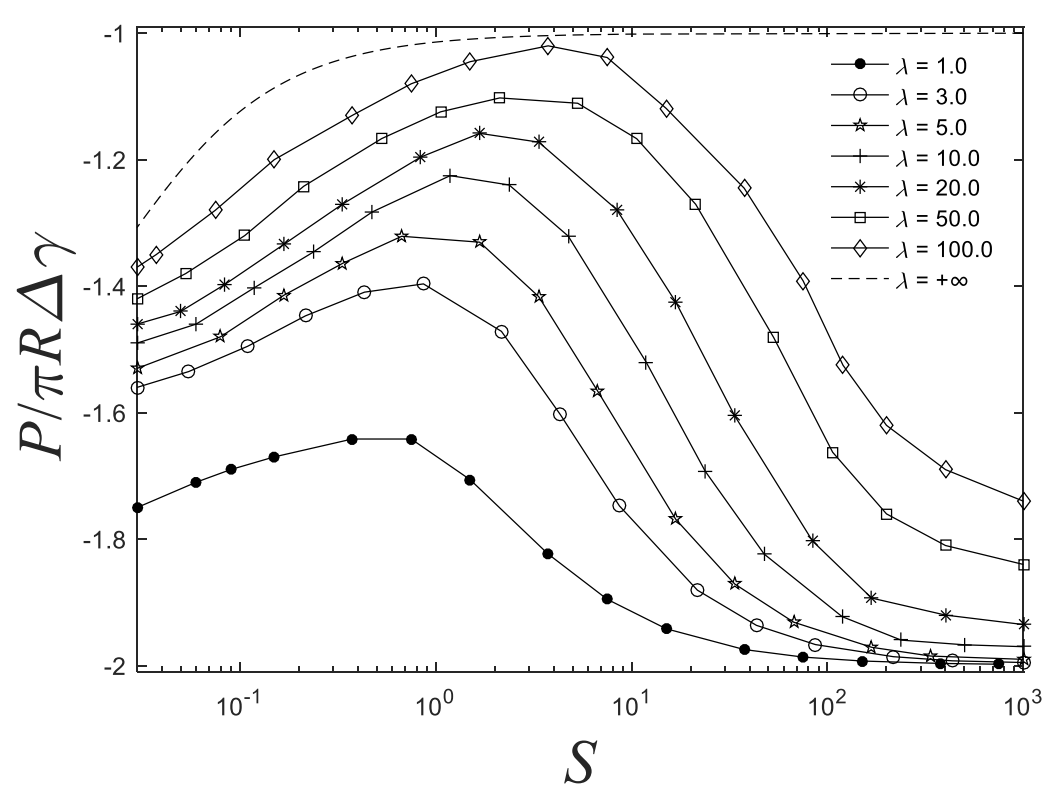

Fig. 7. Pull-off force as a function of the parameter $S$ under different fixed adhesion parameters.

From Figs. 5 and 6, it is clear that there is a non-monotonic trend of the pull-off force versus the parameter $S$. The absolute value of the pull-off force decreases first and then increases as $S$ increases. In Fig. 7, we provide a straightforward illustration of the effect of the parameter $S$ on the pull-forces with different adhesion parameters. Without a surface tension $(S=0)$ and with large adhesion parameters (e.g. $\lambda>5$ ), Maugis (1992) showed that the pull-off force is only slightly lower than 1.5. However, given the same value of adhesion parameters, the existence of a surface tension can increase the pull-off force higher than -1.5 , as shown in Fig. 7 .

As $\lambda$ increases, the non-monotonic dependence of the pull-off force on $S$ becomes significant. For each pull-off force versus $S$ curve, there is a peak point. After going through this point from left to right, the value of the pull-off force dramatically decreases to -2 (Note the scale of abscissa is no longer even). With the parameter $\lambda$ increasing, this point keeps moving toward up and right direction, and asymptotically approaches approaching the point $(+\infty,-1)$. Therefore, we assume that the peak 
point will coincides with the point $(+\infty,-1)$ as the adhesion parameter tends toward infinity, as shown by the dashed curve in Fig. 7. In other words, given an infinite adhesion parameter $\lambda$, the pull-off force increases from -1.5 to -1 monotonically as the parameter $S$ increases from zero to infinity, which is in accordance with the conclusion given by Hui et al. (2015). In his study, a single dimensionless parameter

$$
\omega=\frac{\Gamma}{\left(\frac{9 \pi \Delta \gamma \mu^{2} R^{2}}{4}\right)^{\frac{1}{3}}}(32)
$$

is defined to determine the relation between indentation force (displacement) and contact radius (the Tabor parameter $\mu$ in Eq. (32) can be replaced with $\lambda$, by recalling that $\lambda=1.157 \mu$ ). Their theory reduces to the JKR model and Young-Dupre equation in the limits of small and large $\omega$, respectively. According to the definition of $S$, it is not hard to find

$$
S=\left[6(1-v)^{2}\right]^{\frac{1}{3}} \omega
$$

where $v$ is the Poisson's ratio. Hui et al. (2015) showed that the pull-off force increases from -1.5 to -1 as $\omega$ increases from zero to infinity. In their study, the intermolecular forces are consider as short-range (in fact, as delta function), which means the Tabor parameter (equivalently adhesion parameter if one recalls $\lambda=1.157 \mu$ ) tends toward infinity. Since $S$ is proportional to $\omega$ according to Eq. (33), large $\omega$ corresponds to high $S$, and therefore his conclusion coincides with the dashed curve shown in Fig. 7.

However, all the curves except the dashed curves in Fig. 7 indicate that as long as the adhesion parameter $\lambda$ is finite, the pull-force will decrease to -2 as $S$ tends toward infinity, which is illustrated as follows. As long as the adhesion parameter is finite, the elastic modulus of the half-plane is finite, i.e. the half-plane behaviors as a solid. In this sense, infinite $S$ could be treated as an elastic halfplane covered with a rigid membrane, and thus the pull-force is -2 according to the Bradley model. However, as $\lambda \rightarrow+\infty$, the elastic modulus of the half-plane is infinitesimal as if it behaviors as liquid. In this regard, it is appropriate to treat the contact behavior by using the Young Dupre equation, and thus the pull-off force is -1 (Hui et al., 2015; Long et al., 2016). In conclusion, with finite adhesion parameter $\lambda$, the pull-off force transits between the M-D model and Bradley model, whereas with infinite adhesion parameter $\lambda$, the pull-off force transits between the JKR model and the YoungDupre law. In fact, the general transitions between these four extremes are analogous to our previous study (Zhu and $\mathrm{Xu}, 2018$ ), which explores the effect of surface tension on the adhesive contact behavior where the interaction forces obey the Lennard-Jones potential law. In this regard, we could presumably predict that with surface tension, the transitions of the adhesive contact behaviors between the four extreme models are independent on the exact form of intermolecular interaction potential.

\subsection{Pressure distributions within the contact area}




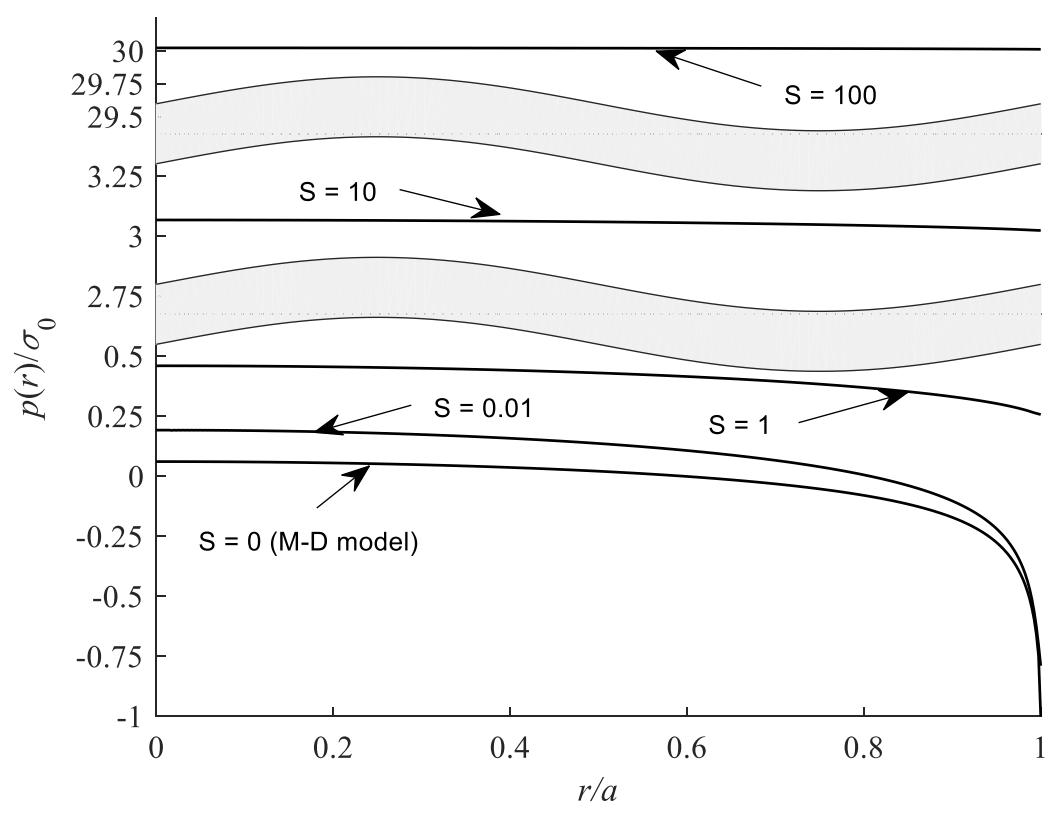

Fig. 8. Distribution of pressure on the contact area corresponding to zero force, with $\lambda=5$.

The pressure distribution is of great interest in the study of contact behavior. Fig. 8 shows the pressure distribution in the contact area normalized by $\sigma_{0}$ (absolute value of the tensile stress in Dugdale laws) corresponding to different $S$ values with $\lambda$ fixed $(\lambda=5)$, under zero load. It can be seen that a larger $S$ will result in a higher distribution of pressure, and as $S$ decreases to zero, the pressure distribution approaches the classic M-D model as expected. With a high value of $S$ (e.g. $S$ $=100$ ), the pressure within the contact region is almost uniformly distributed, however this uniformity weakens as $S$ reduces: the local pressure decreases monotonically as the radial coordinate of the point concerned increases. It is worth our attention that in the present model, the distribution of pressure exhibits a discontinuity on the contact periphery $(r=a)$ which has been implied in a previous study (Long and Wang, 2013) investigating the effect of surface tension on the nonadhesive contact between a rigid sphere and an elastic half-plane with surface tension. Moreover, it is found that the discontinuity weakens as $S$ decreases, and disappears with the absence of surface tension (i.e. $S=0$ ), whose pressure distribution overlaps with the traditional M-D model in Fig. 8, as expected. 


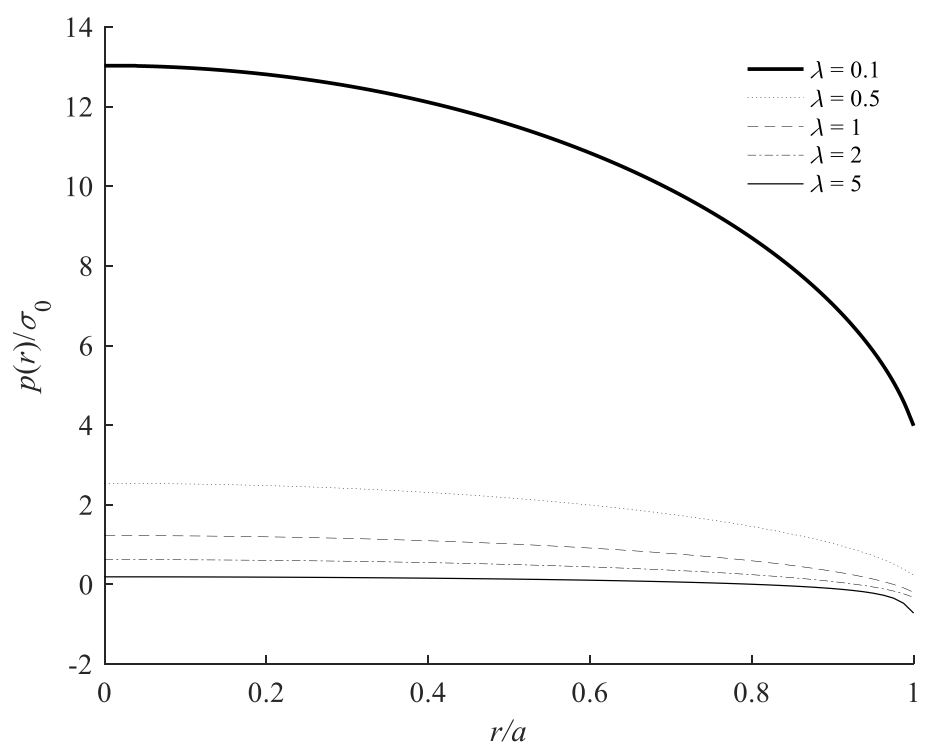

Fig. 9. Distribution of pressure on the contact area corresponding to zero force, with $S=0.1$.

Fig. 9 presents the distribution of normalized pressure with a fixed $S=0.1$ for different values of $\lambda$. There is no overlap between two distribution curves corresponding to two $\lambda$ values, with lower $\lambda$ corresponding to higher distribution of pressure. With a small value of $\lambda$ (e.g. $\lambda=0.1$ ), the pressure covers a large range of value, from approx. 13 in the contact center to approx. 4 on the contact edge. However, the pressure distribution tends to be more even as $\lambda$ increases, as indicated by the thin solid curve in Fig. 9 which corresponds to $\lambda=5$. The discontinuity of pressure is also observed on the contact edge $(r=a)$, and higher $\lambda$ tends to weaken the discontinuity. Moreover, besides the two parameters $\lambda$ and $S$, it is worth our attention that the applied load will also affect the discontinuity of the pressure on the contact edge. As shown in Fig. 10, it is observed that when both $\lambda$ and $S$ are fixed, higher applied load is apt to induce more significant discontinuity on the contact edge.

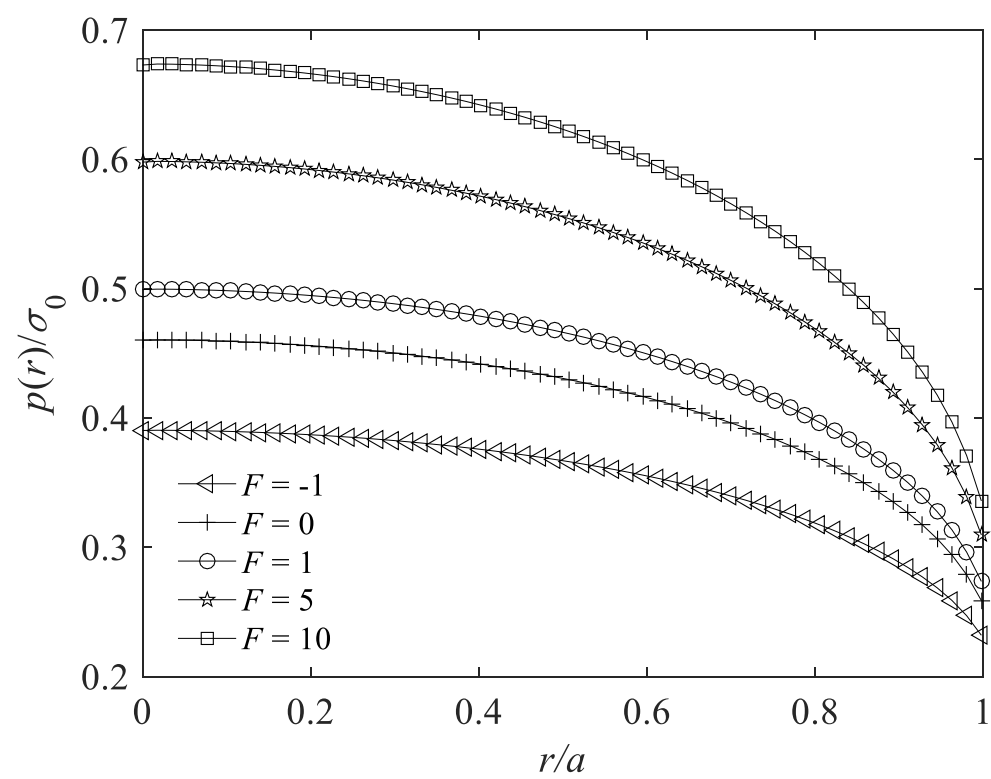

Fig. 10. Distribution of pressure on the contact area with different loads where $F$ denotes the load $P$ scaled by $\pi \Delta \gamma R$, 
with $\lambda=5, S=1$.

\section{Conclusions}

Based on the elastic solution of a concentrated force on a half-plane with surface tension and the adhesive interactions obeying Dugdale law, we develop a Maugis-Dugdale model with existence of surface tension. Analogous to the traditional M-D model, the general results transit from ST-DMT to ST-JKR model as the adhesion parameter $\lambda$ increases with a fixed $S$. Although the existence of surface tension significantly affects the pull-off force in the ST-JKR model, it has negligible effect on the pull-off force in the ST-DMT model. With fixed adhesion parameter $\lambda$ of finite magnitude, there is a non-monotonic dependence of the pull-off force on the parameter $S$. With a finite adhesion parameter $\lambda$, the pull-off force transits from the M-D model to Bradley model as $S$ increases from infinitesimal to infinity, while with an infinite $\lambda$, the pull-off force transits from JKR model to the Young-Dupre law with increasing $S$. The transitions between these four models are akin to our previous study ( $\mathrm{Zhu}$ and $\mathrm{Xu}, 2018$ ) which considers that the intermolecular forces obey the LennardJones potential law. This resemblance inspires us to nervily suppose that the general results of the aforementioned four extreme models are independent of the exact form of the intermolecular interaction potential. This independence also exists in the traditional situation (i.e. without surface tension), that is the general results of the two extreme models (JKR and DMT models) are shown to be independent of the exact form of the intermolecular interaction potential (Muller et al., 1983). Finally, we find that there was discontinuity on the contact perimeter, in terms of contact pressure. The combination of high $S$, low $\lambda$ and high load (or penetration) would correspond to significant existence of the pressure discontinuity.

\section{Acknowledgements}

The authors would like to acknowledge the funding support to Dr Xinyao Zhu as a postdoctoral research fellow from the EU Horizon 2020 (645239) project.

\section{Appendix A. Settlement of the singularity in function $G_{0}(x, y)$}

If $x=y$, Eq. (21a) could be rewritten as

$$
G_{0}(x, x)=\int_{\theta=0}^{\pi} \psi_{0}\left[2 x \sin \left(\frac{\theta}{2}\right)\right] d \theta \text { (A.1) }
$$

If $\theta \rightarrow 0^{+}$, one has

$$
\left.\psi_{0}\left[2 x \sin \left(\frac{\theta}{2}\right)\right] \approx-Y_{0}\left[2 x \sin \left(\frac{\theta}{2}\right)\right] \approx-\frac{2}{\pi}\left\{\ln \left[x \sin \left(\frac{\theta}{2}\right)\right]+\gamma\right\} \text { (А. } 2\right)
$$

where $\gamma$ denotes the Euler-Mascheroni constant, i.e. $\gamma=0.5772156649 \ldots$. The singular onedimensional integral in Eq. (A.1) could be transformed into non-singular two-dimensional integrals by virtue of the identities (Feng, 2000):

$$
\int_{x=0}^{1} f(x) \ln (x) d x=-\int_{x=0}^{1} \int_{y=0}^{1} f(x y) d x d y
$$

where $f(x)$ is a well-behaving function of $x$ on $0 \leq x \leq 1$.

\section{Appendix B. Settlement of the singularity in function $G_{1}(x, y, z)$}

The singularity of the function $G_{1}(x, y, z)$ exhibits only when and only when $x=y$ and $\theta \rightarrow 0$ in Eq. (21b). Herewith, we rewrite Eq. $(21 b)$ as 


$$
G_{1}(x, x, z)=x \int_{\theta=0}^{\pi} \psi_{1}\left(\frac{2 x \sin \frac{\theta}{2}}{z}\right) \sin \frac{\theta}{2} d \theta
$$

As $\theta \rightarrow 0$, one has

$$
x \psi_{1}\left(\frac{2 x \sin \frac{\theta}{2}}{z}\right) \sin \frac{\theta}{2} \rightarrow x\left[-\frac{2}{\pi}-Y_{1}\left(\frac{2 x \sin \frac{\theta}{2}}{z}\right)\right] \sin \frac{\theta}{2}
$$

Noting that when $x \rightarrow 0$

$$
Y_{1}(x) \rightarrow-\frac{\Gamma(1)}{\pi}\left(\frac{2}{x}\right)(\text { B. } 3)
$$

one finally has

$$
\lim _{\theta \rightarrow 0} x \psi_{1}\left(\frac{2 x \sin \frac{\theta}{2}}{z}\right) \sin \frac{\theta}{2}=\frac{\mathrm{z}}{\pi}(\mathrm{B} .4)
$$

\section{References}

Ardito, R., Frangi, A., Rizzini, F., Corigliano, A., 2015. Evaluation of adhesion in microsystems using equivalent rough surfaces modeled with spherical caps. Eur. J. Mech. A/Solids 57, 121-131.

Bian, J., Wang, G., Feng, X., 2012. Atomistic calculations of surface energy of spherical copper surfaces. Acta Mech. Solida Sin. 25 557-561.

Bradley, R.S., 1932. The cohesive force between solid surfaces and the surface energy of solids. Phil. Mag. 13, 853-862.

Cammarata, R.C., 1994. Surface and interface stress effects in thin films. Prog. Surf. Sci. 46, 1-38.

Chen, S.H., Yao, Y., 2014. Elastic Theory of Nanomaterials Based on Surface-Energy Density. J. Appl. Mech. 81,121002.

Chen, T.Y., Chiu, M.S., Weng, C.N., 2006. Derivation of the generalized Young-Laplace equation of curved interfaces in nanoscaled solids. J. Appl. Phys. 100, 074308.

Chen, W.Q., Zhang, Ch., 2010. Anti-plane shear Green's functions for an isotropic elastic half-space with a material surface. Int. J. Solids Struct. 47, 1641-1650.

Derjaguin, B.V., Muller, V.M., Toporov, Y.P., 1975. Effect of contact deformations on the adhesion of particles. J. Coll. Interface Sci. 53, 314-326.

Ding, Y., Niu, X.R., Wang, G.F., 2015. Elastic compression of nanoparticles with surface energy. J. Phys. D: Appl. Phys. 48, 485303.

Feng, J.Q., 2000. Contact behavior of spherical elastic particles: a computational study of particle adhesion and deformations. Colloids Surf. A, Physicochem. Eng. Asp. 172, 175-198.

Gao, X., Hao, F., Fang, D.N., Huang, Z.P., 2013. Boussinesq problem with the surface effect and its application to contact mechanics at the nanoscale. Int. J. Solids Struct. 50, 2620-2630. 
Gao, X., Hao, F., Huang, Z.P., Fang, D.N., 2014. Mechanics of adhesive contact at the nanoscale: the effect of surface stress. Int. J. Solids Struct. 51, 566-574.

Greenwood, J.A., 1997. Adhesion of elastic spheres. Proc. R. Soc. Lond. A 453, 1277-1297.

Gurtin, M.E., Murdoch, A.I., 1975. A continuum theory of elastic material surfaces. Arch. Ration. Mech. Anal. 57, 291-323.

Gurtin, M.E., Weissmuller, J., Larche, F., 1998. A general theory of curved deformable interfaces in solids at equilibrium. Philos. Mag. A 78, 1093-1109.

Hajji, M.A., 1978. Indentation of a membrane on an elastic half space. J. Appl. Mech. 45, 320-324.

He, L., Lim, C., 2006. Surface Green function for a soft elastic half-space: influence of surface stress. Int. J. Solids Struct. 43, 132-143.

Hertz, H., 1882. On the contact between elastic bodies. J. Reine Angew. Math. 92, 156-171.

Huang, G.Y., Yu, S.W., 2007. Effect of surface elasticity on the interaction between steps. J. Appl. Mech. 74, 821-823.

Huang, Z.P., Wang, J., 2006. A theory of hyperelasticity of multi-phase media with surface/interface energy effect. Acta Mech. 182, 195-210.

Hui, C.Y., Liu, T.S., Salez, T., Raphael, E., Jagota, A., 2015. Indentation of a rigid sphere into an elastic substrate with surface tension and adhesion. Proc. R. Soc. A 471, 20140727.

Johnson, K.L., Kendall, K., Roberts, A.D., 1971. Surface energy and the contact of elastic solids. Proc. R. Soc. Lond. A 324, 301-313.

Koguchi, H., 2004a. Contact and Adhesion Analysis Considering a Variation of Surface Stresses: 1st Report, A Comparison of Fundamental Theory and Hertz Theory. Jpn. Soc. Mech. Eng., A 70, 289-297.

Koguchi, H., 2004b. Contact and Adhesion Analysis Considering a Variation of Surface Stresses: 2nd Report, A Comparison of The Present Theory and JKR Theory. Jpn. Soc. Mech. Eng., A 70, $1332-1340$.

Koguchi, H., 2008. Surface Green function with surface stresses and surface elasticity using Stroh's formalism. J. Appl. Mech. 75, 061014-061025.

Koguchi, H., Hayashi, T., 2009. Contact Analysis for Anisotropic Materials Considering Surface Stresses and Surface Elasticity. Trans. Jpn. Soc. Mech. Eng., A 75, 1029-1036. 
Long, J.M., Wang, G.F., 2013. Effects of surface tension on axisymmetric Hertzian contact problem. Mech. Mater. 56, 65-70.

Long, J.M., Wang, G.F., Feng, X.Q., Yu, S.W., 2016. Effects of surface tension on the adhesive contact between a hard sphere and a soft substrate. Int. J. Solids Struct. 84, 133-138.

Long, J.M., Ding, Y., Yuan, W.K., Chen, W., Wang, G.F., 2017. General relations of indentations on solids with surface tension. ASME J. Appl. Mech. 84, 051007.

Maugis, D., 1992. Adhesion of spheres: The JKR-DMT transition using a Dugdale model. J. Colloid Interface Sci.150, 243-269.

Miller, R.E., Shenoy, V.B., 2000. Size-dependent elastic properties of nanosized structural elements. Nanotechnology. 11, 139-147.

Mogilevskaya, S.G., Pyatigorets, A.V., Crouch, S.L., 2011. Green function for the problem of a plane containing a circular hole with surface effects. J. Appl. Mech. 78, 021008.

Muller, V.M., Yushchenko, V.S., Derjaguin, B.V., 1983. General theoretical consideration of the influence of surface forces on contact deformations and the reciprocal adhesion of elastic spherical particles, J. Coll. Interface Sci. 92, 92-101.

Shenoy, V.B., 2002. Size-dependent rigidities of nanosized torsional elements. Int. J. Solids Struct. 39, 4039-4052.

Shenoy, V.B., 2005. Atomistic calculations of elastic properties of metallic fcc crystal surfaces. Phys. Rev. B 71, 094104.

Salez, T., Benzaquen, M., Raphaël, É., 2013. From adhesion to wetting of a soft particle. Soft Matter. 9, 10699-10704.

Style, R.W., Hyland, C., Boltyanskiy, R., Wettlaufer, J.S., Dufresne, E.R., 2013. Surface tension and contact with soft elastic solids. Nat. Commun. 4, 2728-2733.

Tabor, D., 1977. Surface forces and surface interactions. J. Colloid Interface Sci. 58, 2-13.

Wang, G.F., Feng, X.Q., 2007. Effects of surface stresses on contact problems at nanoscale. J. Appl. Phys. 101, 013510.

Yang, F.Q., 2004. Size-dependent effective modulus of elastic composite materials: spherical nanocavities at dilute concentrations. J. Appl. Phys. 95, 3516-3520.

Zhao, X.J., Rajapakse, R.K.N.D., 2009. Analytical solutions for a surface-loaded isotropic elastic 
layer with surface energy effects. Int. J. Eng. Sci. 47, 1433-1444.

Zhu, X.Y., Xu W., 2018. Effect of surface tension on the behavior of adhesive contact based on Lennard-Jones potential law. J. Mech. Phys. Solids. 111, 170-183. 